OPEN ACCESS

Edited by:

Oren Levy,

Bar-Ilan University, Israel

Reviewed by:

Riccardo Rodolfo Metalpa

IRD Nouméa,

New Caledonia

Alexander Venn

Center Scientific de Monaco, Monaco

*Correspondence: Gretchen Goodbody-Gringley ggoodbody@reefresearch.org Tali Mass

tmass@univ.haifa.ac.il

Specialty section

This article was submitted to Coral Reef Research

a section of the journal

Frontiers in Marine Science

Received: 19 November 2019

Accepted: 09 January 2020

Published: 24 January 2020

Citation:

Scucchia F, Nativ H, Neder M, Goodbody-Gringley G and Mass T (2020) Physiological Characteristics of Stylophora pistillata Larvae Across a Depth Gradient.

Front. Mar. Sci. 7:13

doi: 10.3389/fmars.2020.00013

\section{Physiological Characteristics of Stylophora pistillata Larvae Across a Depth Gradient}

\author{
Federica Scucchia',2, Hagai Nativ ${ }^{1}$, Maayan Neder ${ }^{1,2}$, Gretchen Goodbody-Gringley ${ }^{3 *}$ \\ and Tali Mass ${ }^{1 *}$ \\ ${ }^{1}$ Department of Marine Biology, Leon H. Charney School of Marine Sciences, University of Haifa, Haifa, Israel, ${ }^{2}$ The \\ Interuniversity Institute of Marine Sciences, Eilat, Israel, ${ }^{3}$ Bermuda Institute of Ocean Sciences, Central Caribbean Marine \\ Institute, St. George's, Bermuda
}

Depth related parameters, specifically light, affect different aspects of corals physiology, including fluorescence. Green fluorescence protein (GFP)-like pigments found in many coral species have been suggested to serve a variety of functions, including photo-protection and photo-enhancement. Using fluorescence imaging and molecular analysis, we further investigated the role of these proteins on the physiology of the coral Stylophora pistillata and its algal partners. Fluorescence was found to differ significantly between depths for larvae and adult colonies. Larvae from the shallow reef presented a higher GFP expression and a greater fluorescence intensity compared to the larvae from the mesophotic reef, reflecting the elevated need for photo-protection against high light levels characteristic of the shallow reef, thus supporting the "sunscreen" hypothesis. Additionally, given the lower but still occurring protein expression under non-damaging low light conditions, our results suggest that GFP-like proteins might act to regulate the amount of photosynthetically usable light for the benefit of the symbiotic algae. Moreover, we propose that the differences in GFP expression and green fluorescence between shallow and deep larvae indicate that the GFPs within coral larvae might serve to attract and retain different symbiont clades, increasing the chances of survival when encountering new environments.

Keywords: fluorescence, corals, GFP, horizontal acquisition, mesophotic reef, Symbiodinium

\section{INTRODUCTION}

Scleractinian corals are the foundation organisms of tropical coral reefs, providing the physical structure and habitat that supports the world most diverse and productive biological communities on Earth. They owe their success, in part, to their symbiotic relationship with photosynthetic endosymbiotic dinoflagellate algae in the family Symbiodinaceae, that supply the coral host with sufficient photosynthetic products, in particular lipids and carbohydrates, to support the hosts' metabolism, growth, reproduction, and survival (Hoegh-Guldberg, 1999; Davy et al., 2012; Allemand and Furla, 2018). During photosynthesis Symbiodinaceae rely on light as the energy source, thereby restricting the distribution of many coral species to the photic zone. However, corals are able to persist and form reef structures in deeper waters despite limited light availability (Ezzat et al., 2017). Referred to as mesophotic reefs, these light-dependent coral ecosystems (Armstrong et al., 2006; Hinderstein et al., 2010) range in depth from $30 \mathrm{~m}$ to almost $150 \mathrm{~m}$ in some areas 
with particularly clear waters (Bongaerts et al., 2010). Given the documented influence of light on the photosynthetic features of Symbiodinium sp., light characteristics are one of the main factors influencing the depth-zonation of symbiotic corals (Nir et al., 2011). Across their vertical distribution, from high-lightdominated shallow areas to low-light-dominated mesophotic reefs, variations in light intensity induce both the coral and the dinoflagellate partner to present adaptive traits and photophysiological modifications (Bongaerts et al., 2011; Ezzat et al., 2017; Smith et al., 2017).

Corals also possess pigments related to the green fluorescence proteins (GFPs) of Aequorea victoria (Tsien, 1998) that have been suggested as important players in corals acclimatization and adaptation to changing light conditions with depth (Salih et al., 2000; Dove et al., 2001). In shallow-water corals these pigments are suggested to serve as sunscreens (Salih et al., 2000; Ben-Zvi et al., 2015), reducing light stress of the algal symbiont through the absorption of high energy and potentially harmful photons and re-emitting light with lower energy (Roth and Deheyn, 2013). However, intensely fluorescent corals have been observed in lowlight, deep-water environments, suggesting a different role for these GFP-like proteins in mesophotic reefs (Smith et al., 2017). In particular, these pigments have been hypothesized to have a photo-enhancement function through the transformation of the incident light into wavelengths of peak absorption by the algae photosynthetic pigments (Kawaguti, 1969; Schlichter and Fricke, 1990). To date, the functions of GFP-like proteins in corals remains controversial and ambiguous, with other proposed functions for these proteins including attraction of free-living Symbiodinium sp. (Hollingsworth et al., 2005), oxidative stress response (Bou-Abdallah et al., 2006), camouflage (Matz et al., 2006) and immune response (Palmer et al., 2009). Regardless, the connection between coral fluorescence and abiotic factors indicates that these proteins may play a major role in coral interactions with the physical environment (Roth et al., 2013).

Abiotic factors that vary with depth also affect patterns of coral recruitment, with light representing one of the main elements influencing pre- and post-settlement survival and behavior (Turner et al., 2018). The resilience of coral reefs to increasing threats such as climate change hinges on successful recruitment, where phenotypic diversity and plasticity of larvae may enable a wide range of tolerances and the variation necessary for natural selection to act (Roth et al., 2013). It is critical, therefore, to obtain a comprehensive understanding of coral larval physiological adaptation to environmental variation, since their effective settlement provides the basis for the future of the reef (Aranda et al., 2011).

In the present study, we examined the characteristics of early life stages of the depth generalist coral Stylophora pistillata, Esper, 1797, along a depth gradient in the Gulf of Eilat, Red Sea. S. pistillata is one of the most abundant hermatypic coral species in the Gulf of Eilat (Loya, 1972). This hermaphroditic brooder typically releases planula larvae between December and July (Grinblat et al., 2018). Here, we examined key physiological features of $S$. pistillata larvae, analyzing differential photophysiology and GFP-like protein characteristics between shallow and mesophotic planulae, to explore the potential connection between these proteins and different light environments, and to discuss their possible role in the physiology of corals and of their algal partners.

\section{MATERIALS AND METHODS}

\section{Sample Collection}

Larvae traps were made using a $160 \mu \mathrm{m}$ plankton net with a plastic collection container attached to the top. Larvae of the stony coral S. pistillata were collected from randomly selected colonies on the reef adjacent to the Interuniversity Institute of Marine Sciences (IUI, $29^{\circ} 30^{\prime} 06.0^{\prime \prime} \mathrm{N} 34^{\circ} 54^{\prime} 58.3^{\prime \prime} \mathrm{E}$ ) in the Gulf of Eilat (Israel), both from the shallow reef (depth of 3-6 m) and from the mesophotic reef (depth of 40-45 m) under a special permit from the Israeli Natural Parks Authority (Figure 1).

Collection traps were placed on random 15 colonies for each depth shortly before sunset and retrieved the following morning; this process was repeated for three nights near the full moon of April 2019, following peak releases (Shlesinger et al., 1998). Light intensity and seawater temperature of the sampling sites were measured using HOBO Water Temp Pro data loggers (Onset Computers). For each sample, half of the planulae collected were immediately placed in Petri dishes with seawater for larval size and fluorescence imaging analysis, the remaining larvae were immediately snap-frozen in liquid nitrogen and stored with TRI reagent (Life Technologies) at $-80 \mathrm{C}$ for DNA and RNA extraction.

\section{Larval Size Image Analyses}

Larvae from samples pooled by depth were randomly subsampled ( $n=36$, shallow; $n=32$ mesophotic) and digitally photographed from a side view (elliptical in shape) using a Dino-Lite digital microscope under a $3 \times$ magnification. Larval volume was determined by measuring the length and width of the longitudinal and transverse axes in Image J and applying the equation for volume of an elliptical sphere, $V=\frac{4}{3} \pi a b^{2}$, where $a$ is $1 / 2$ width and $b$ is $1 / 2$ length (Isomura and Nishihira, 2001). Larval volume was square-root transformed in order to meet the assumptions of normality (Shapiro-Wilk's test) and homogeneity (Levene's test) and compared by depth using an Analysis of Variance (ANOVA) followed by a Tukey's HSD post hoc test performed in RStudio v1.1.423 (R Development Team) using the above function in the package car (Fox et al., 2019).

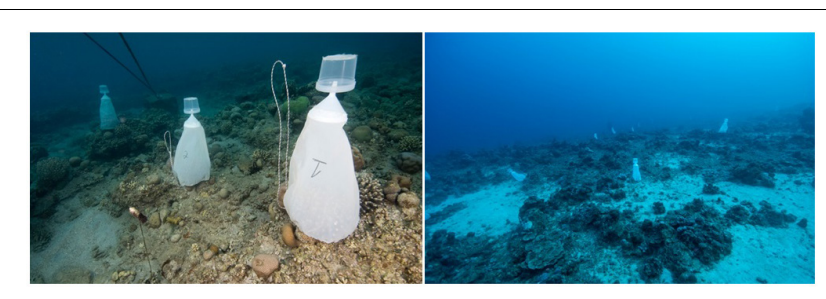

FIGURE 1 | Images of larval collection traps on adult Stylophora pistillata colonies in the Red Sea at 3-6 (left) and 40-45 m (right) depths. 


\section{RNA Extraction and cDNA Synthesis}

Total RNA was extracted from pools of 10-15 larvae per sample using the Invitrogen PureLink RNA micro kit (Thermo Fisher Scientific, United States) according to the manufacturers' protocol. DNAase treatment was performed within the RNA extraction procedures according to the manufacturers' instructions (Thermo Fisher Scientific, United States). RNA concentration and quality were confirmed using a NanoDrop 2000 (Thermo Fisher Scientific, United States) and electrophoresis using a 1\% agarose gel under denaturing conditions. The RNA integrity was assessed based on clear $28 \mathrm{~S}$ and $18 \mathrm{~S}$ ribosomal RNA bands on a $1 \%$ agarose gel using electrophoresis. First strand cDNA for each sample was synthesized from $150 \mathrm{ng}$ total RNA using the Thermo Scientific RevertAid First Strand cDNA Synthesis kit following the manufacturers' protocol (Thermo Fisher Scientific, United States).

\section{Quantification of GFP Expression}

Expression levels of GFP proteins were assessed in coral larvae using quantitative real-time polymerase chain reaction (qRTPCR). GFP primers (5'-GAAATGAGCCTCAAAGGCAAC- $3^{\prime}$ forward and 5'-GTTGGTTCCCAGCCAAGA-3' reverse) were selected according to Grinblat et al. (2018), as well as the two internal control genes primers, b-actin and adenosylhomocysteinase (AdoHcyase).

Each reaction contained $2 \times$ SYBR Green PCR Master Mix (Thermo Fisher Scientific, United States), $0.2 \mu \mathrm{M}$ specific primers and cDNA of each sample. Amplification reactions were carried out with an initial denaturation step of $95^{\circ} \mathrm{C}$, followed by 40 cycles of $95^{\circ} \mathrm{C}$ for $3 \mathrm{~s}$ and $60^{\circ} \mathrm{C}$ for $30 \mathrm{~s}$, a dissociation step of $95^{\circ} \mathrm{C}$ for $15 \mathrm{~s}$, annealing step at $60^{\circ} \mathrm{C}$ for $1 \mathrm{~min}$ and then melting back to $95^{\circ} \mathrm{C}$ over $20 \mathrm{~min}$ (StepOnePlus ${ }^{\mathrm{TM}}$ Real-Time PCR System, Applied Biosystems, United States).

\section{Symbiodinium ITS2 Analyses}

Eight larvae from the shallow and eight larvae from the mesophotic reef where pooled together in four different subsamples (two per depth range). Genomic DNA was extracted from the four subsamples using the Wizard genomic DNA purification kit (Promega Corporation, United States) according to the manufacturers' protocol. The internal transcribed spacer (ITS2) region of Symbiodinium rDNA was amplified using Symbiodinium-specific primers taken from Arif et al. (2014). The amplified ITS2 fragments were separated with electrophoresis using a $1 \%$ agarose gel under denaturing conditions. Ten $\mu \mathrm{L}$ of the above PCR products were sent to HyLab (Hy Laboratories Ltd., Israel) where they were subjected to a second PCR using the Access Array tag for Illumina primers (Fluidigm Corporation, United States). This second PCR added the Index and adaptor sequences required for sequencing on the Illumina system. The samples were then purified using AMPure XP beads (Beckman Coulter Inc., United States) and the concentration was determined by Qubit (Thermo Fisher Scientific, United States). The samples were pooled together and sequenced on the Illumina Miseq using a v2-500 cycle kit to generate $250 \times 2$, paired-end reads. The data was de-multiplexed by the Illumina software, and the de-multiplexed FASTQ files were further analyzed. The resulting OTUs sequences were aligned with ClustalX (Larkin et al., 2007) and blasted on GenBank ${ }^{1}$.

\section{In situ Imaging System}

In situ adult corals were imaged during the day when the polyps were opened by modifying the wide band fluorescence imaging system, FluorIS (Treibitz et al., 2015) for fluorescence imaging in situ. The systems consist of a Nikon D850 camera with a Nikon $35 \mathrm{~mm} 1.8$ lens, housed within a Nauticam housing. In the FluorIS system, the camera was modified by replacing the IR filter over its sensor with a clear filter that transmits the entire light spectrum; the sensor is sensitive thoroughly $300-1200 \mathrm{~nm}$. A custom-made $25 \times 25 \mathrm{~cm}$ quadrat was used to enable rapid imaging of the same area. Using the above methodology, the fluorescence images were captured using the FluorIS as previously described (Treibitz et al., 2015; Zweifler et al., 2017). Ambient light (Iambient) was subtracted from the daytime fluorescence (Iday) images in MATLAB in order to receive a fluorescence image similar to nighttime fluorescence image (Fstrobes) using the equation Fstrobes = Iday - Iambient (Zweifler et al., 2017). Mean GFP and chlorophyll fluorescence intensities were determined in ImageJ (Schneider et al., 2012) delimiting and selecting the area of the images covered by the colonies and calculating the mean gray value, which was estimated separately for the green and the red channel. The average intensities $(N=3)$ were calculated and expressed as percentage change \pm relative standard error.

\section{Fluorescence Microscopy and Image Analyses}

Coral planulae were imaged while not being retracted using an Inverted Phase Contrast Fluorescent Microscope (Nikon Eclipse Ti, Melville, NY, United States). Each sample was observed with a DS-Ri2 camera using two single fluorescence channels, red (emission $590 \mathrm{~nm}$ ) and green (emission $515 \mathrm{~nm}$ ), that were additionally merged together. Exposure and gain settings per each channel were kept constant between shallow and deep larvae (150 ms exposure and 1.1 gain for the red channel, $20 \mathrm{~ms}$ exposure and 1 gain for the green channel). All images were acquired with the Nikon Nis-Elements software (Nikon Instruments, Melville, NY, United States). Mean GFP and chlorophyll fluorescence intensities were determined using the mean gray value in ImageJ (Schneider et al., 2012), delimiting and selecting the area within the larvae edges. The average intensities $(N=3)$ were calculated and expressed as percentage change \pm relative standard error.

\section{Data Analysis}

Univariate comparisons of $\mathrm{qPCR}$ data relative to the GFP expression were performed using the non-parametric one-way ANOVA (Kruskal-Wallis test), followed by the Mann-Whitney $U$-test $(P<0.05)$, after verifying the deviations from parametric

\footnotetext{
${ }^{1}$ http://www.ncbi.nlm.nih.gov/BLAST
} 
ANOVA assumptions (Normality: Shapiro-Wilk's test; equal variance: Bartlett's test and Brown-Forsythe test). The GraphPad Prism 8 software (GraphPad Inc.) was used to perform all the statistical tests and to create the graphs. Data are presented as means (fold changes) \pm standard errors.

Similarities between GFPs gene sequences were determined using the percent identity matrix calculated after the alignment with ClustalX. The alignment figure was created using Jalview 2.10.5 (Waterhouse et al., 2009).

Symbiodinium OTUs resulted from the ITS2 analyses were aligned using ClustalX and the percent identity matrix was used to determine similarities between OTUs of the same clade.

\section{RESULTS}

\section{Larval Size Image Analyses}

Larvae were collected from 45 different colonies at each depth during three nights near the full moon of April 2019. Forty colonies spawned at the shallow water while only nine colonies spawned at the deep water during these nights, suggesting a delayed spawning peak at the mesophotic depth. A total of 600 and 100 larvae were collected from the shallow and deep-water colonies, respectively.

The mean larval volume of the shallow larvae was found to be significantly higher compared to the deep larvae by approximately two-fold (Figure 2, $P<0.0001$ ). Light intensity, measured with the HOBO Water Temp Pro data loggers, ranged from 6,200 to 228 lux at 6 and $45 \mathrm{~m}$, respectively.

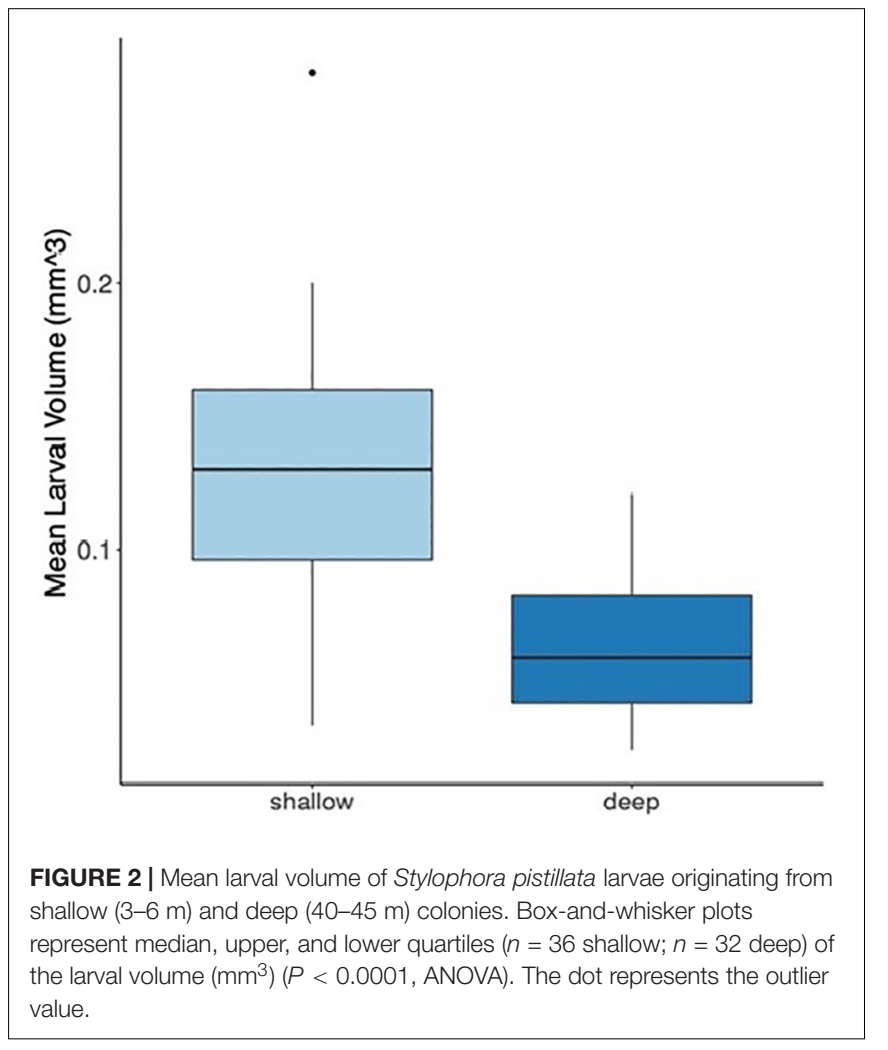

\section{Symbiodinium Clade Analyses}

A total of 1,19,399 ITS2 high quality sequence reads (mean length $=320 \mathrm{bp}$ ) were obtained from 4 total samples (two samples per depth range), each containing 4 coral larvae. A large proportion of the filtered OTUs were non -Symbiodinium organisms that were prevalent mostly in the water and as part of the coral holobiome. After the removal of non -Symbiodinium sequences and of singletons (OUTs with 1 sequence read) only Symbiodinium OTUs remained and were clustered into clade A and clade C, at $97 \%$ similarity (sequences were blasted on GenBank, see text footnote 1).

The deep larvae contained symbiont consortia dominated by Symbiodinium clade C (99.8\%) with a small percentage representing Symbiodinium clade A (0.2\%). In contrast, the symbiont consortia present in shallow larvae was dominated by Symbiodinium clade A $(99.9 \%)$ with a small percentage of Symbiodinium clade C (0.1\%) present.

\section{Fluorescence in situ and Microscopy Analysis}

Coral fluorescence of both GFP and chlorophyll-a were visibly different between adult $S$. pistillata colonies from $6 \mathrm{~m}$ (Figures 3A-C) and those from $45 \mathrm{~m}$ (Figures 3D,E). GFP fluorescence intensity was on average $65.4 \% \pm 6.6(N=3)$ lower for the mesophotic adult corals compared to the shallow adult corals (Figures 3B,E), while Symbiodinium chlorophyll intensity was on average $54.6 \% \pm 8.4(N=3)$ lower for the shallow corals compared to the deep corals (Figures 3C,F). In addition, the GFP fluorescence appeared evenly distributed along the length of all the branches in the coral at $6 \mathrm{~m}$ (Figure 3B inset), but it was more intense at the branch tips of the coral at $45 \mathrm{~m}$ (Figure $3 \mathrm{E}$ inset). A similar pattern of GFP and chlorophyll fluorescence was also found for shallow and mesophotic larvae as to the adult colonies at the same depth, with an average $54.5 \% \pm 7.9(N=3)$ lower GFP fluorescence in the deep larvae compared to the shallow larvae (Figures 4B,E), and an average $27.7 \% \pm 2.3(N=3)$ lower chlorophyll intensity in the shallow larvae compared to the deep larvae (Figures 4C,F). Furthermore, the distribution of GFP within the larvae was also similar to the adults, where shallow larvae displayed a uniform fluorescence pattern and mesophotic larvae had higher GFP intensity around the mouth and at the aboral epidermis (Figures 4A,D).

\section{Quantification of GFP Expression}

Specific expression of the S. pistillata GFP-like chromoprotein (Accession number: DQ206398.1; Grinblat et al., 2018) was evaluated in S. pistillata larvae from the shallow and mesophotic reef through comparisons of mRNA expression and was found to be significantly lower for mesophotic larvae (0.8-fold downregulation) compared to shallow larvae (Figure 5, $P<0,005$ ).

The expression pattern of the GFP-like chromoprotein observed in this study corresponds with the expression patterns analyzed for several GFP transcripts in adult colonies of S. pistillata from different depths $(5,10,20,30,50$, and $60 \mathrm{~m})$ in the Gulf of Eilat (Malik et al., 2020) (Supplementary Figure S1). In particular, a correspondence was found between the GFP-like 

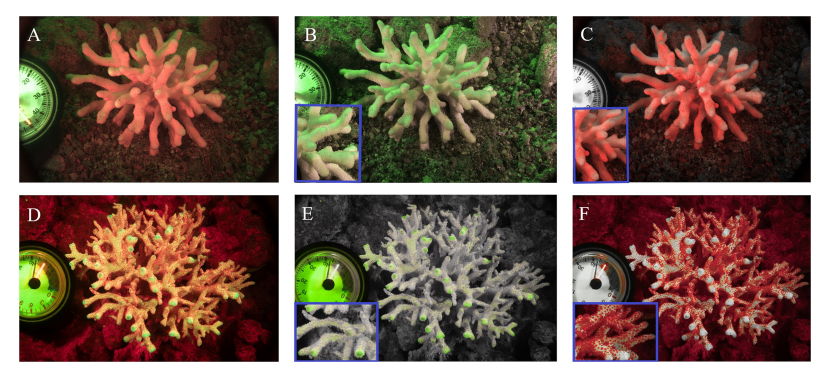

FIGURE 3 | Fluorescence camera images of S. pistillata adults from the shallow reef $(\mathbf{A}-\mathbf{C})$ and from the mesophotic reef (D-F). Green fluorescence indicates GFPs fluorescence (B,E), red fluorescence indicates the chlorophyl fluorescence of Symbiodinium (C,F), overlap of the two channels $(\mathbf{A}, \mathbf{D})$. Blue rectangular inserts represent a $4 \times$ magnification of the relative image (B,C,E,F).
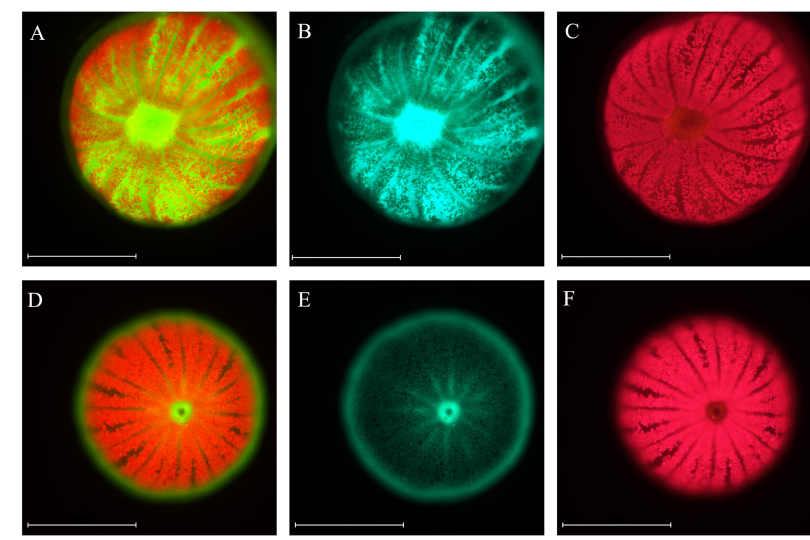

FIGURE 4 | Microscopic images of $S$. pistillata larvae from the shallow reef (A-C) and from the mesophotic reef (D-F). Green fluorescence indicates GFPs fluorescence (B,E), red fluorescence indicates the chlorophyll fluorescence of Symbiodinium (C,F), overlap of the two channels $(\mathbf{A}, \mathbf{D})$. Microscope exposure and gain settings are identical in both shallow and mesophotic larvae; chlorophyll exposure is $20 \mathrm{~ms}$ and gain 1, GFP exposure is $150 \mathrm{~ms}$ and gain 1.1. Magnification: $10 \times$. Scale bar: $500 \mu \mathrm{m}$.

chromoprotein gene analyzed for the larvae and the GFP2 gene examined in the adults (95\% identity) (Figure 6).

\section{DISCUSSION}

Resilience of coral reefs under rapid environmental change heavily relies on larval survival and effective settlement, thus it is crucial to thoroughly comprehend coral larval physiological adaptation to environmental features. Many coral species have been shown to differ in morphology and physiology along a broad depth gradient, and these modification have often been indicated as the result of phenotypic plasticity in response to the variation of environmental conditions with depth (Bruno and Edmunds, 1997; Einbinder et al., 2009; Goodbody-Gringley and Waletich, 2018). In this study, we observed clear patterns of depth-related physiological characteristics in S. pistillata

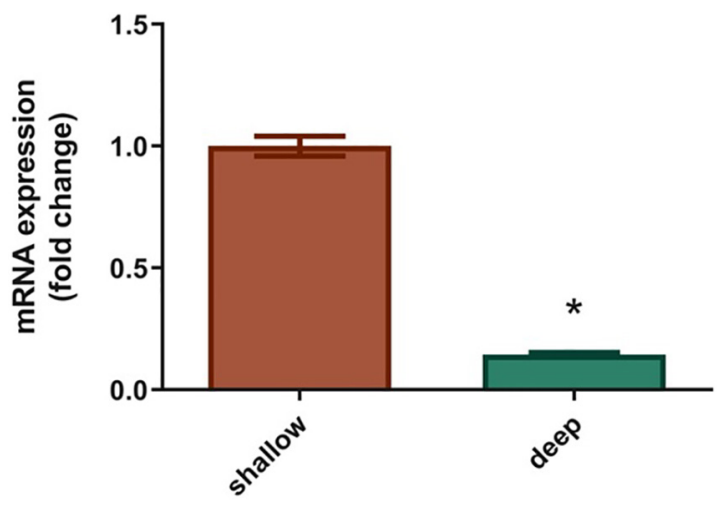

FIGURE 5 | Expression of GFP-like chromoprotein in Stylophora pistillata shallow and deep larvae. Levels of GFP are expressed as relative variation (fold change) of deep larvae GFP relative to shallow larvae GFP (means $\pm \mathrm{SEMs} ; N=6$ ). ${ }^{\star} P<0.05$ vs. ctr (Mann-Whytney $U$-test).

larvae from the shallow and the mesophotic reef, particularly looking at the differences in larval size, in the association with different symbiont assemblages and in the GFP expression and fluorescence.

We found that the shallow larvae are significantly larger than the deep ones (Figure 2), and we attributed this difference to the constraints of the light environment in the parental habitat. In fact, it has been previously shown that coral larvae deriving from high light adapted parents were larger than larvae belonging to low light adapted adults (Roth et al., 2013). Specifically, higher light availability in shallow reef conditions may result in higher energetic reserves, enabling larvae from the shallow reef to obtain a larger size compared to deep larvae, which might not be able to support a similar size range given the limited light energy available on the mesophotic reef (Lesser et al., 2018; Kahng et al., 2019).

Although energetic content of the larvae was not addressed in this study, comparisons between parental depth and the amount of maternally derived lipids within the resulting larvae that are used as endogenous source of energy may further elucidate the role of light availability on larval size (Woodley et al., 2015; Rivest et al., 2017). Differences in the density of Symbiodinacaea cells and chlorophyll concentrations within larvae can also affect the amount of translocated metabolites from the symbiotic algae, which can act as a significant sources of energy for coral larvae (Richmond, 1987; Harii et al., 2010).

Previous studies have shown that deep and low light adapted corals contain more chlorophyll per Symbiodinium sp. than those adapted to high light environments (Falkowski and Dubinsky, 1981; Mass et al., 2007; Cohen and Dubinsky, 2015; Smith et al., 2017). Thus, the greater concentration of photosynthetic pigments observed in this study in the adults (Figure 3F) and in the deep larvae via differential fluorescence (Figure 4F), may be attributed to the maximization of the algae light harvesting capacity through increased concentration of photosynthetic pigments within the symbionts, as formerly reported in S. pistillata by Titlyanov et al. (2001), representing 


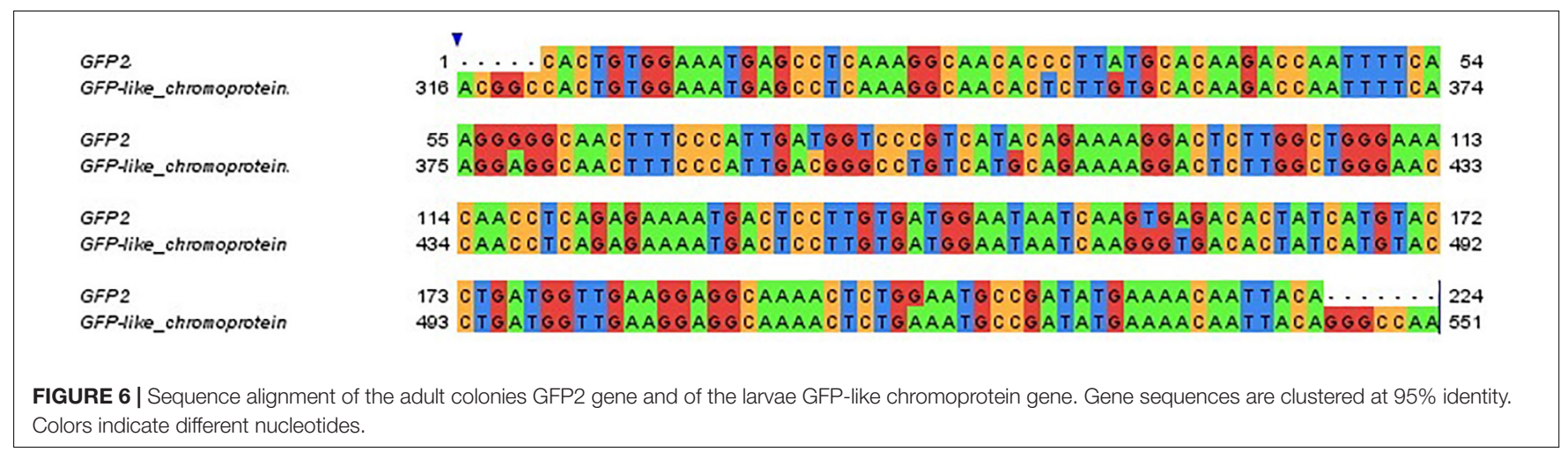

a habitat-specific adaptation to light-limited mesophotic depths (Winters et al., 2009).

The differential patterns of representation by the two Symbiodinium clades detected in this study in the larvae are similar to the ones found in adult $S$. pistillata colonies by Malik et al. (2020), where shallow colonies harbored predominantly clade A, and deep colonies harbored predominantly clade C.

Roughly $90 \%$ of brooding scleractinian species vertically transmit Symbiodinium, one of these being S. pistillata (Baird et al., 2009) and, within these species, if the same parent or different parents host different symbionts, the progeny can inherit all or none of the parental symbionts (Byler et al., 2013; Reich et al., 2017). Given the similarity in Symbiodinium consortia between S. pistillata adult colonies (Malik et al., 2020) and the larvae, this study provides evidence to further support vertical acquisition of both primary and secondary symbiont clades, as previously hypothesized for S. pistillata (Byler et al., 2013) and for other scleractinian species (Van Oppen, 2004; Reich et al., 2017). However, horizontal acquisition during development may also occur, where S. pistillata might be able to attract and acquire specific symbiont clades from the environment during larval and juvenile stages (Reich et al., 2017).

The ability of individual juveniles to gain and maintain novel symbionts through horizontal acquisition might provide a crucial mechanism to increase the chances of survival in a new environment during dispersal at the larval stage (Byler et al., 2013) and, at the same time, the inheritance of symbionts from the parents would assure the supply of photosynthetic products during the delicate initial larval stages. Previous studies have shown that different clades of Symbiodinium have differential preferences for the light intensity required for photosynthesis and that they are differently affected by the host internal light environment variation, which can be achieved through the modulation of the GFPs fluorescence intensity (Ezzat et al., 2017; Quigley et al., 2018; Aihara et al., 2019). Since the light intensity necessary to attract specific symbionts likely differs with light spectra and depth (Aihara et al., 2019), coral larvae from shallow and deep reefs potentially generate diverse internal light environments, displaying different GFP fluorescence depending on the preferred association with different Symbiodinium clades (Yuyama and Higuchi, 2014). Thus, the different intensities of GFP fluorescence detected in this study between shallow and mesophotic larvae (Figures 4B,E) suggests that host-modulated fluorescence may represent a mechanism to attract specific symbiont types with unique physiologies. Moreover, the concentrated GFPs fluorescence around the mouth could further denote the ability of the larvae to attract and capture free-living Symbiodinium sp. via consumption, as suggested by Hollingsworth et al. (2005).

The depth ranges chosen for this study (6 and $45 \mathrm{~m}$ ) represent two distinct habitats where corals endure either high intensity or limited light levels. Corals possess specific physiological characteristics to cope with such varying light environments, one of these being the expression of the GFP proteins, which has been indicated as a photoprotective mechanism in shallow water corals (Salih et al., 2000, 2006) and as a way to increase light availability for the symbiotic algae in deep water corals (Dove et al., 2001). In this study the higher GFP expression levels (Figure 5) and the higher intensity of GFP fluorescence found in the shallow larvae (Figure 4B) is consistent with the role of these proteins as UVR screeners. In fact, coral larvae are particularly susceptible to UVR (Aranda et al., 2011) and they receive a higher amount of solar radiation in shallow-water areas compared to the mesophotic reef, therefore mechanisms to dissipate high-energy light wavelengths are crucial to provide photo-protection to both the coral and the symbiotic algae.

In the adult colonies, we observed a higher fluorescence intensity in the shallow reef (Figure 3B) compared to the mesophotic reef (Figure 3E); furthermore, the majority of the examined GFP transcripts showed a significant down-regulation with depth, with a marked differential expression between 10 and 20-60 m depth (Supplementary Figure S1), in accordance with the photoprotective role of the GFP proteins (Salih et al., 2000) and the up-regulation of oxidative stress response and DNArepair genes, which correlate with higher photosynthesis activity of the symbiont in shallow water (Malik et al., 2020).

Previous studies have shown that GFPs have a robust photo-acclimation response, modulating the coral internal light environment and potentially influencing the physiology of the symbiotic algae and of the coral itself (Roth et al., 2010; Lyndby et al., 2016). Results of the present study support the light screening model whereby GFPs in shallow-water corals protect against the damages caused by elevated UVR levels, but it can also disclose the possible role of these proteins in deep-water 
corals. For example, this study found that GFPs are still being expressed under non-damaging low light conditions (Figure 5) and that certain GFPs are more highly expressed in the deep reef compared to the shallow (GFP5, Supplementary Figure S1). Additionally, some GFPs were found to be up-regulated at deeper depths $(60 \mathrm{~m}$, Supplementary Figure S1) relative to shallower areas (20 and $30 \mathrm{~m}$, Supplementary Figure S1). Taken together, these results suggest that these proteins have different roles depending on the depth (Ben-Zvi et al., 2015). In particular, in deeper waters GFPs might act to regulate the amount of photosynthetically available light, creating an optimal environment for photosynthesis (Dove et al., 2001; Quigley et al., 2018). Specifically, GFPs in the mesophotic corals could be actively regulating of the amount of photosynthetically available light through transformation of blue-green wavelengths into longer wavelengths via fluorescence in the coral tissue, thereby enhancing photosynthetic performance of the symbionts (Salih et al., 2006; Smith et al., 2017; Quigley et al., 2018). Such modifications of internal wavelengths would alter the light spectrum and increase internal light intensity surrounding the symbiotic dinoflagellate cells in order to overcome limited light availability on mesophotic reefs. This mechanism, together with the differential preferences for light intensity required for photosynthesis of different Symbiodinium clades, might explain the relative differences in GFP expression and in the hosted Symbiodinium clades observed in this study between the shallow and the deep reef in S. pistillata larvae and adults.

\section{CONCLUSION}

The data presented here highlight that depth-related parameters, specifically light, play a major role in the photo-physiology of corals and their associated Symbiodinium, and corroborate the notion that GFPs act as UVR screeners in high light environments, as supported by the higher GFP expression and the greater fluorescence intensity observed in S. pistillata shallow larvae, compared to the deep ones. Moreover, the lower but still occurring GFP expression under non-damaging low light conditions underlines another possible function of these proteins, such that they might act to regulate the amount of photosynthetically available light in order to optimize the internal light field for the benefit of the symbiotic algae.

Additionally, the observed differences in the GFP expression and the green fluorescence between the shallow and deep larvae, suggests that GFPs within coral larvae may act to attract and retain different symbionts clades with specific physiologies, as well as enhance photosynthesis through the creation of divergent internal light environments, increasing the chances of survival when encountering new environments. This work potentially provides the bases for future studies investigating the functional

\section{REFERENCES}

Aihara, Y., Maruyama, S., Baird, A. H., Iguchi, A., Takahashi, S., and Minagawa, J. (2019). Green fluorescence from cnidarian hosts attracts symbiotic algae. Proc. Natl. Acad. Sci. U.S.A. 116, 2118-2123. doi: 10.1073/pnas.1812257116 significance of the association between coral GFPs fluorescence and the symbiont assemblages, especially focusing on a crucial life stage for corals, such as the larval phase.

\section{DATA AVAILABILITY STATEMENT}

The raw data supporting the conclusion of this article will be made available by the authors, without undue reservation, to any qualified researcher.

\section{AUTHOR CONTRIBUTIONS}

FS, MN, HN, GG-G, and TM carried out the experiments, participated in the design of the study, contributed to the writing and improving of the manuscript, and approved its final version. FS, GG-G, and TM participated in the data analysis and wrote the manuscript. FS carried out the molecular analysis. FS, MN, and GG-G carried out the microscopy imaging and the image analysis. HN carried out the in situ imaging.

\section{FUNDING}

This work was supported by the Israel Science Foundation (Grant 312/15), United States-Israel Binational Science Foundation (BSF; Grant \# 2016321), the European Research Council (ERC; Grant \# 755876), and the ASSEMBLE Plus consortium for an access grant (ref. SR16022018108e1) to the Interuniversity Institute for Marine Sciences in Eilat.

\section{ACKNOWLEDGMENTS}

We thank the technical staff of the Moris Kahn Marine Research Station for their invaluable help. We thank Alex Chequer, Shai Eindinder, and Stephan Martinez for their assistance with technical diving field work. We thank the technical staff of the Interuniversity Institute of Marine Sciences for the invaluable help with the field study. We thank Opher Bar-Natan for helping with image processing. This study was performed in accordance with regulations and guidelines set by the Israel Nature and National Park Protection Authority.

\section{SUPPLEMENTARY MATERIAL}

The Supplementary Material for this article can be found online at: https://www.frontiersin.org/articles/10.3389/fmars. 2020.00013/full\#supplementary-material

Allemand, D., and Furla, P. (2018). How does an animal behave like a plant? Physiological and molecular adaptations of zooxanthellae and their hosts to symbiosis. Comptes. Rendus. Biol. 341, 276-280. doi: 10.1016/j.crvi.2018.03.007 Aranda, M., Banaszak, A. T., Bayer, T., Luyten, J. R., Medina, M., and Voolstra, C. R. (2011). Differential sensitivity of coral larvae to natural levels of ultraviolet 
radiation during the onset of larval competence. Mol. Ecol. 20, 2955-2972. doi: 10.1111/j.1365-294x.2011.05153.x

Arif, C., Daniels, C., Bayer, T., Banguera-Hinestroza, E., Barbrook, A., Howe, C. J., et al. (2014). Assessing Symbiodinium diversity in scleractinian corals via nextgeneration sequencing-based genotyping of the ITS2 rDNA region. Mol. Ecol. 23, 4418-4433. doi: 10.1111/mec.12869

Armstrong, R. A., Singh, H., Torres, J., Nemeth, R. S., Can, A., Roman, C., et al. (2006). Characterizing the deep insular shelf coral reef habitat of the Hind Bank marine conservation district (US Virgin Islands) using the Seabed autonomous underwater vehicle. Continent. Shelf Res. 26, 194-205. doi: 10.1016/j.csr.2005. 10.004

Baird, A. H., Guest, J. R., and Willis, B. L. (2009). Systematic and biogeographical patterns in the reproductive biology of scleractinian corals. Ann. Rev. Ecol. Evol. Syst. 40, 551-571. doi: 10.1146/annurev.ecolsys.110308.120220

Ben-Zvi, O., Eyal, G., and Loya, Y. (2015). Light-dependent fluorescence in the coral Galaxea fascicularis. Hydrobiologia 759, 15-26. doi: 10.1007/s10750-0142063-6

Bongaerts, P., Ridgway, T., Sampayo, E. M., and Hoegh-Guldberg, O. (2010). Assessing the 'deep reef refugia' hypothesis: focus on Caribbean reefs. Coral Reefs 29, 309-327. doi: 10.1007/s00338-009-0581-x

Bongaerts, P., Riginos, C., Hay, K. B., van Oppen, M. J., Hoegh-Guldberg, O., and Dove, S. (2011). Adaptive divergence in a scleractinian coral: physiological adaptation of Seriatopora hystrix to shallow and deep reef habitats. BMC Evol. Biol. 11:303. doi: 10.1186/1471-2148-11-303

Bou-Abdallah, F., Chasteen, N. D., and Lesser, M. P. (2006). Quenching of superoxide radicals by green fluorescent protein. Biochim. Biophys. Acta Gen. Sub. 1760, 1690-1695. doi: 10.1016/j.bbagen.2006.08.014

Bruno, J. F., and Edmunds, P. J. (1997). Clonal variation for phenotypic plasticity in the coral Madracis mirabilis. Ecology 78, 2177-2190. doi: 10.2307/2265954

Byler, K. A., Carmi-Veal, M., Fine, M., and Goulet, T. L. (2013). Multiple symbiont acquisition strategies as an adaptive mechanism in the coral Stylophora pistillata. PLoS One 8:e59596. doi: 10.1371/journal.pone.0059596

Cohen, I., and Dubinsky, Z. (2015). Long term photoacclimation responses of the coral Stylophora pistillata to reciprocal deep to shallow transplantation: photosynthesis and calcification. Front. Mar. Sci. 2:45. doi: 10.3389/fmars.2015. 00045

Davy, S. K., Allemand, D., and Weis, V. M. (2012). Cell biology of cnidariandinoflagellate symbiosis. Microbiol. Mol. Biol. Rev. 76, 229-261. doi: 10.1128/ mmbr.05014-11

Dove, S. G., Hoegh-Guldberg, O., and Ranganathan, S. (2001). Major colour patterns of reef-building corals are due to a family of GFP-like proteins. Coral Reefs 19, 197-204. doi: 10.1007/pl00006956

Einbinder, S., Mass, T., Brokovich, E., Dubinsky, Z., Erez, J., and Tchernov, D. (2009). Changes in morphology and diet of the coral Stylophora pistillata along a depth gradient. Mar. Ecol. Prog. Ser. 381, 167-174. doi: 10.3354/meps 07908

Ezzat, L., Fine, M., Maguer, J. F., Grover, R., and Ferrier-Pagès, C. (2017). Carbon and nitrogen acquisition in shallow and deep holobionts of the scleractinian coral S. pistillata. Front. Mar. Sci. 4:102. doi: 10.3389/fmars.2017.00102

Falkowski, P. G., and Dubinsky, Z. (1981). Light-shade adaptation of Stylophora pistillata, a hermatypic coral from the Gulf of Eilat. Nature 289:172. doi: 10 . 1038/289172a0

Fox, J., Weisberg, S., Friendly, M., Hong, J., Andersen, R., Firth, D., et al. (2019). Package 'Effects'. Available at: https://cran.r-project.org/web/packages/effects/ index.html

Goodbody-Gringley, G., and Waletich, J. (2018). Morphological plasticity of the depth generalist coral, Montastraea cavernosa, on mesophotic reefs in Bermuda. Ecology 99, 1688-1690. doi: 10.1002/ecy.2232

Grinblat, M., Fine, M., Tikochinski, Y., and Loya, Y. (2018). Stylophora pistillata in the Red Sea demonstrate higher GFP fluorescence under ocean acidification conditions. Coral Reefs 37, 309-320. doi: 10.1007/s00338-018-1659-0

Harii, S., Yamamoto, M., and Hoegh-Guldberg, O. (2010). The relative contribution of dinoflagellate photosynthesis and stored lipids to the survivorship of symbiotic larvae of the reef-building corals. Mar. Biol. 157, 1215-1224. doi: 10.1007/s00227-010-1401-0

Hinderstein, L. M., Marr, J. C. A., Martinez, F. A., Dowgiallo, M. J., Puglise, K. A., Pyle, R. L., et al. (2010). Theme section on "Mesophotic coral ecosystems: characterization, ecology, and management. Coral Reefs 29, 247-251. doi: 10. 1007/s00338-010-0614-5

Hoegh-Guldberg, O. (1999). Climate change, coral bleaching and the future of the world's coral reefs. Mar. Freshw. Res. 50, 839-866. doi: 10.1071/MF99078

Hollingsworth, L. L., Kinzie, R. A., Lewis, T. D., Krupp, D. A., and Leong, J. A. C. (2005). Phototaxis of motile zooxanthellae to green light may facilitate symbiont capture by coral larvae. Coral Reefs 24, 523-523. doi: 10.1007/s00338-0050063-8

Isomura, N., and Nishihira, M. (2001). Size variation of planulae and its effect on the lifetime of planulae in three pocilloporid corals. Coral Reefs 20, 309-315. doi: 10.1007/s003380100180

Kahng, S. E., Akkaynak, D., Shlesinger, T., Hochberg, E. J., Wiedenmann, J., Tamir, R., et al. (2019). Light, temperature, photosynthesis, heterotrophy, and the lower depth limits of mesophotic coral ecosystems. Mesophot. Coral Ecosyst. 12, 801-828. doi: 10.1007/978-3-319-92735-0_42

Kawaguti, S. (1969). Effect of the green fluorescent pigment on the productivity of the reef corals. Micronesica 5:121.

Larkin, M. A., Blackshields, G., Brown, N. P., Chenna, R., McGettigan, P. A., McWilliam, H., et al. (2007). Clustal W and Clustal X version 2.0. Bioinformatics 23, 2947-2948. doi: 10.1093/bioinformatics/btm404

Lesser, M. P., Slattery, M., and Mobley, C. D. (2018). Biodiversity and functional ecology of mesophotic coral reefs. Ann. Rev. Ecol. Evol. Syst. 49, 49-71. doi: 10.1146/annurev-ecolsys-110617-062423

Loya, Y. (1972). Community structure and species diversity of hermatypic corals at Eilat Red Sea. Mar. Biol. 13, 100-123. doi: 10.1007/bf00366561

Lyndby, N. H., Kühl, M., and Wangpraseurt, D. (2016). Heat generation and light scattering of green fluorescent protein-like pigments in coral tissue. Sci. Rep. 6:26599. doi: 10.1038/srep26599

Malik, A., Einbinder, S., Martinez, S., Tchernov, D., Haviv, S., Almuly, R., et al. (2020). Molecular and skeletal fingerprints of scleractinian coral biomineralization: from the sea surface to mesophotic depths. Acta Biomater. (in press). doi: 10.1016/j.actbio.2020.01.010

Mass, T., Einbinder, S., Brokovich, E., Shashar, N., Vago, R., Erez, J., et al. (2007). Photoacclimation of Stylophora pistillata to light extremes: metabolism and calcification. Mar. Ecol. Prog. Ser. 334, 93-102. doi: 10.3354/meps334093

Matz, M. V., Marshall, N. J., and Vorobyev, M. (2006). Symposium-in-print: green fluorescent protein and homologs-are corals colorful. Photochem. Photobiol. 82, 345-350.

Nir, O., Gruber, D. F., Einbinder, S., Kark, S., and Tchernov, D. (2011). Changes in scleractinian coral Seriatopora hystrix morphology and its endocellular Symbiodinium characteristics along a bathymetric gradient from shallow to mesophotic reef. Coral Reefs 30:1089. doi: 10.1007/s00338-011-0801-z

Palmer, C. V., Roth, M. S., and Gates, R. D. (2009). Red fluorescent protein responsible for pigmentation in trematode-infected Porites compressa tissues. Biol. Bull. 216, 68-74. doi: 10.1086/bblv216n1p68

Quigley, K. M., Strader, M. E., and Matz, M. V. (2018). Relationship between Acropora millepora juvenile fluorescence and composition of newly established Symbiodinium assemblage. PeerJ 6:e5022. doi: 10.1101/271155

Reich, H. G., Robertson, D. L., and Goodbody-Gringley, G. (2017). Do the shuffle: changes in Symbiodinium consortia throughout juvenile coral development. PLoS One 12:e0171768. doi: 10.1371/journal.pone.0171768

Richmond, R. H. (1987). Energetics, competency, and long-distance dispersal of planula larvae of the coral Pocillopora damicornis. Mar. Biol. 93, 527-533. doi: $10.1007 /$ bf00392790

Rivest, E. B., Chen, C. S., Fan, T. Y., Li, H. H., and Hofmann, G. E. (2017). Lipid consumption in coral larvae differs among sites: a consideration of environmental history in a global ocean change scenario. Proc. R. Soc. B Biol. Sci. 284:20162825. doi: 10.1098/rspb.2016.2825

Roth, M. S., and Deheyn, D. D. (2013). Effects of cold stress and heat stress on coral fluorescence in reef-building corals. Sci. Rep. 3:1421. doi: 10.1038/srep01421

Roth, M. S., Fan, T. Y., and Deheyn, D. D. (2013). Life history changes in coral fluorescence and the effects of light intensity on larval physiology and settlement in Seriatopora hystrix. PLoS One 8:e59476. doi: 10.1371/journal. pone.0059476

Roth, M. S., Latz, M. I., Goericke, R., and Deheyn, D. D. (2010). Green fluorescent protein regulation in the coral Acropora yongei during photoacclimation. Eur. J. Exp. Biol. 213, 3644-3655. doi: 10.1242/jeb.040881 
Salih, A., Cox, G., Szymczak, R., Coles, S. L., Baird, A. H., Dunstan, A., et al. (2006). "The role of host-based color and fluorescent pigments in photoprotection and in reducing bleaching stress in corals," in Proceedings of the 10th International Coral Reef Symposium. 2006, Okinawa.

Salih, A., Larkum, A., Cox, G., Kühl, M., and Hoegh-Guldberg, O. (2000). Fluorescent pigments in corals are photoprotective. Nature 408:850. doi: 10 . $1038 / 35048564$

Schlichter, D., and Fricke, H. W. (1990). Coral host improves photosynthesis of endosymbiotic algae. Naturwissenschaften 77, 447-450. doi: 10.1007/ bf01135950

Schneider, C. A., Rasband, W. S., and Eliceiri, K. W. (2012). NIH Image to ImageJ: 25 years of image analysis. Nat. Methods 9:671. doi: 10.1038/nmeth.2089

Shlesinger, Y., Goulet, T. L., and Loya, Y. (1998). Reproductive patterns of scleractinian corals in the northern Red Sea. Mar. Biol. 132, 691-701. doi: 10.1007/s002270050433

Smith, E. G., D'angelo, C., Sharon, Y., Tchernov, D., and Wiedenmann, J. (2017). Acclimatization of symbiotic corals to mesophotic light environments through wavelength transformation by fluorescent protein pigments. Proc. R. Soc. B Biol. Sci. 284:20170320. doi: 10.1098/rspb.2017.0320

Titlyanov, E. A., Titlyanova, T. V., Yamazato, K., and Van Woesik, R. (2001). Photoacclimation dynamics of the coral Stylophora pistillata to low and extremely low light. J. Exp. Mar. Biol. Ecol. 263, 211-225. doi: 10.1016/s0022-0981(01)003 09-304

Treibitz, T., Neal, B. P., Kline, D. I., Beijbom, O., Roberts, P. L., Mitchell, B. G., et al. (2015). Wide field-of-view fluorescence imaging of coral reefs. Sci. Rep. 5:7694. doi: $10.1038 /$ srep07694

Tsien, R. Y. (1998). The green fluorescent protein. Annu. Rev. Biochem. 67, 509-544.

Turner, J. A., Thomson, D. P., Cresswell, A. K., Trapon, M., and Babcock, R. C. (2018). Depth-related patterns in coral recruitment across a shallow to mesophotic gradient. Coral Reefs 37, 711-722. doi: 10.1007/s00338-018-16961698
Van Oppen, M. J. (2004). Mode of zooxanthella transmission does not affect zooxanthella diversity in acroporid corals. Mar. Biol. 144, 1-7. doi: 10.1007/ s00227-003-1187-4

Waterhouse, A. M., Procter, J. B., Martin, D. M., Clamp, M., and Barton, G. J. (2009). Jalview Version 2-a multiple sequence alignment editor and analysis workbench. Bioinformatics 25, 1189-1191. doi: 10.1093/bioinformatics/ btp033

Winters, G., Beer, S., Zvi, B. B., Brickner, I., and Loya, Y. (2009). Spatial and temporal photoacclimation of Stylophora pistillata: zooxanthella size, pigmentation, location and clade. Mar. Ecol. Prog. Ser. 384, 107-119. doi: 10. 3354/meps08036

Woodley, C. M., Downs, C., Bruckner, A. W., Porter, J. W., and Galloway, S. B. eds (2015). Diseases of Coral. Hoboken, NJ: John Wiley \& Sons.

Yuyama, I., and Higuchi, T. (2014). Comparing the effects of symbiotic algae (Symbiodinium) clades $\mathrm{C} 1$ and $\mathrm{D}$ on early growth stages of Acropora tenuis. PLoS One 9:e98999. doi: 10.1371/journal.pone.00 98999

Zweifler, A., Akkaynak, D., Mass, T., and Treibitz, T. (2017). In situ analysis of coral recruits using fluorescence imaging. Front. Mar. Sci. 4:273. doi: 10.3389/fmars. 2017.00273

Conflict of Interest: The authors declare that the research was conducted in the absence of any commercial or financial relationships that could be construed as a potential conflict of interest.

Copyright (c) 2020 Scucchia, Nativ, Neder, Goodbody-Gringley and Mass. This is an open-access article distributed under the terms of the Creative Commons Attribution License (CC BY). The use, distribution or reproduction in other forums is permitted, provided the original author(s) and the copyright owner(s) are credited and that the original publication in this journal is cited, in accordance with accepted academic practice. No use, distribution or reproduction is permitted which does not comply with these terms. 